\title{
Fine structure of unoccupied Ag $d$ states near the Fermi level in Ag and AgPd studied by high-resolution partial Auger yield spectroscopy at the $\mathrm{Ag} L_{3}$ edge
}

\author{
W. Drube, ${ }^{1}$ T. K. Sham, ${ }^{2}$ A. Kravtsova, ${ }^{3}$ and A. V. Soldatov ${ }^{3}$ \\ ${ }^{1}$ Hamburger Synchrotronstrahlungslabor HASYLAB at Deutsches Elektronen-Synchrotron DESY, \\ Notkestr., 85, D-22603 Hamburg, Germany \\ ${ }^{2}$ Department of Chemistry, University of Western Ontario, London N6A 5B7, Canada \\ ${ }^{3}$ Faculty of Physics, Rostov State University, Sorge 5, Rostov-na-Donu 344090, Russia
}

(Received 1 July 2002; published 31 January 2003)

\begin{abstract}
The $\mathrm{Ag} L_{3}$-edge x-ray-absorption fine structure of $\mathrm{Ag}$ metal and $\mathrm{AgPd}$ alloy was measured using the $\mathrm{Ag}$ $L_{3} M_{4,5} M_{4,5}\left({ }^{1} G_{4}\right)$ Auger electron yield. The core-hole lifetime broadening of the spectra could be reduced due to high-energy resolution in the present experimental technique, allowing for the study of the fine details of partial density of states in the conduction band of the samples. The fine structure of the partial density of states around the Fermi level was also studied in a theoretical analysis using a full multiple-scattering technique. The calculated absorption structure is in good agreement with the experimental data. The combined results indicate a reduction of the density of $\mathrm{Ag} \boldsymbol{d}$ and $\boldsymbol{s}$ states at and several $\mathrm{eV}$ above the Fermi level upon the AgPd alloy formation compared to the pure Ag metal.
\end{abstract}

DOI: 10.1103/PhysRevB.67.035122

PACS number(s): 78.70.Dm

\section{INTRODUCTION}

The electronic structure of $4 d$ transition-metal (TM) alloys has been extensively studied in the last decade. ${ }^{1}$ Different high-energy spectroscopies were used for the experimental investigation of the electron densities of states. X-ray photoelectron spectroscopy (XPS) and x-ray emission spectroscopy (XES) provide information about the occupied states $^{2}$ while the unoccupied states become accessible in x-ray-absorption spectroscopy (XAS) and bremsstrahlung isochromat spectroscopy (BIS). ${ }^{3}$ For the unoccupied states, the additional advantage of XAS over BIS is its selectivity, i.e., that while BIS spectra reflect the distribution of the total density of states (DOS) XAS probes local (i.e., corresponding to a specific atom type in a given chemical environment) and projected (i.e., having specific symmetry, $s, p, d$, etc.) unoccupied electronic states. ${ }^{3}$

The distribution of electronic states near the Fermi level is one of the key parameters, which determines the physical and chemical properties of the alloys. In the $4 d \mathrm{TM}$ and their alloys the $4 d$ orbitals contribute primarily to the region near the Fermi level ${ }^{1}$ and thus play a crucial role in determining the properties of these solids. The characteristics of these states depend significantly on the occupation of the $4 d$ shell. In particular, the properties of the partially filled and fully filled $4 d$ shell are distinctly different. The DOS of the partially filled $d$ band, e.g., in Pd, crosses the Fermi level with high densities of states at the Fermi level. For nominally $d^{10}$ metals, such as $\mathrm{Ag}$, the top of the $d$ band is well below the Fermi level (about $2 \mathrm{eV}$ in $\mathrm{Ag}$ ). This paper addresses the unoccupied states at and a few $\mathrm{eV}$ above the Fermi level in the $\mathrm{AgPd}$ alloy system where the $\mathrm{Ag}$ and $\mathrm{Pd} 4 d$ shell is fully and partially filled, respectively.

XPS (Refs. 4 and 5) and XES (Ref. 6) have been used previously to study the occupied electronic states of $\mathrm{AgPd}$ alloys. These techniques generally yield information about the occupied DOS, i.e., the valence bands below the Fermi level. In XAS on the other hand, the x-ray-absorption nearedge structures (XANES) at the TM $L_{2,3}$ edges primarily probe the local density of unoccupied $d$ states (the contribution of $s$ states is found to be only about several percent). In the open-shell case, a pronounced resonance ("white line") is generally observed at the $L_{23}$ edges. The analysis of its intensity has been used to study the $4 d$ charge transfer in alloys. ${ }^{7}$ The XANES features at the TM $L_{2,3}$ edge obtained by the conventional total electron yield (TEY) and fluorescence yield (FY) measurements are hampered by the corehole lifetime broadening $\left(2.0 \mathrm{eV}\right.$ for the $\mathrm{Ag} L_{3}$ level $\left.^{8}\right)$. This broadening can be significantly reduced in high-resolution partial yield experiments that monitor the intensity of Auger electrons or fluorescence radiation associated with a narrow decay channel, i.e., a transition to a comparatively long-lived final state. ${ }^{9}$ In addition, the partial yield measurement is element selective and therefore significantly enhances the element contrast, which is especially advantageous in the case of PdAg alloys because of the proximity of the constituents' $L_{2,3}$ edges.

Using the Ag Auger yield, chemical systematic in $\mathrm{AgAu}$ alloys were studied with sublifetime resolution $\mathrm{Ag} L_{3}$ XANES. ${ }^{10}$ This approach is applied here to obtain highresolution $\mathrm{Ag} L_{3}$ XANES of the AgPd alloys. A recent XPS and XANES study of these alloys has been reported by Coulthard et al. ${ }^{5}$ but the absorption spectra were only measured using the TEY, smearing out fine details of the XANES which are revealed in the present study. The present approach is a unique method for the study of the fine structures of $\mathrm{Ag} \boldsymbol{d}$ and $\boldsymbol{s}$ projected density of states in the continuum part of the conduction band at and just above the Fermi level.

The electronic structure of $\mathrm{Ag}_{0.5} \mathrm{Pd}_{0.5}$ has been studied theoretically in detail by Lu et al. ${ }^{11}$ However, they did not provide information on the unoccupied electronic states exceeding $2 \mathrm{eV}$ above $E_{F}$ because of the well-known limitations of the applied plane-wave methods. Also, the calculated total DOS below $E_{F}$ in $\mathrm{Ag}$ metal and $\mathrm{Ag}_{0.5} \mathrm{Pd}_{0.5}$ does not 
exhibit the pronounced double structure observed in the XPS data. ${ }^{5,12}$ A closely related theoretical method has recently been applied ${ }^{13}$ to study the charge transfer effects in AgPd alloys. The calculated $L_{3}$ absorption spectra only extend up to $3 \mathrm{eV}$ above the Fermi level. Within this interval, the shape of the theoretical $\mathrm{Ag} L_{3}$-XANES spectra of $\mathrm{Ag}$ and $\mathrm{AgPd}$ differs significantly from the experimental data obtained previously by TEY, ${ }^{5}$ and in the present study. Therefore it is appropriate to perform a further theoretical study to an extended region above the Fermi level.

\section{EXPERIMENTS AND CALCULATION}

The experiments were performed at the high-flux wiggler beamline BW2 at HASYLAB Hamburg, Germany. The alloys were prepared with stoichiometric amounts of ultrahigh pure metals as described previously. ${ }^{5}$ The samples were cleaned in UHV by Ar sputtering and annealing cycles prior to the measurement. No contaminant was detected in the photoelectron spectra. The kinetic energy of the electrons was selected with a hemispherical electron energy analyzer (Scienta SES 200), the resulting total-energy resolution, i.e., photons and electrons, was $0.7 \mathrm{eV}$ (full width at half maximum). The typical photon flux on the sample was 1 $\times 10^{12} \mathrm{sec}^{-1}$ (spot size $\sim 1 \times 1 \mathrm{~mm}^{2}$ ). The absolute monochromator energy was calibrated by measuring the $\mathrm{Cu}$ $K$-edge absorption edge $(8979 \mathrm{eV})$. The absolute accuracy at the $\mathrm{Ag} L_{3}$ edge is estimated $\pm 0.5 \mathrm{eV}$, the relative uncertainty is smaller than $\pm 0.1 \mathrm{eV}$. Partial Auger yield XANES measurements were made by monitoring the intensity of the ${ }^{1} G_{4}$ Auger line of the $L_{3} M_{4,5} M_{4,5}$ decay at constant kinetic energy within an energy window $\Delta E=1 \mathrm{eV}$.

A complete $\mathrm{Ag} L_{3} M_{4,5} M_{4,5}$ Auger spectrum of AgPd excited with a photon energy $3 \mathrm{eV}$ above the $\mathrm{Ag} L_{3}$ threshold is shown in Fig. 1. The pronounced peaks can be associated with the corresponding final states of the $M_{4,5} M_{4,5}$ final-state multiplet, as indicated in the figure. ${ }^{8}$ The intensity of the most prominent peak, ${ }^{1} G_{4}$, was chosen for the partial yield measurement. The final-state kinetic energy was fixed at the peak center as measured for excitation energy well above threshold, i.e., above the region where energy dispersion due to the resonant Auger Raman effect is significant. In Fig. 2, the corresponding partial yield spectra are shown in an energy range, which also includes the $\mathrm{Pd} L_{2}$ edge. The TEY spectrum (solid line) is in agreement with previous data ${ }^{5}$ and exhibits rather broad XANES structure due to the $L_{3}$ lifetime broadening. Also note the significant contribution of the Pd XANES to the Ag absorption signal. Due to the element selectivity of the Auger yield data (symbols), this background is reduced by a factor of 5. In addition, the $\mathrm{Ag}$ XANES exhibits much sharper structures because of the reduced lifetime broadening. In general, an interpretation of the partial yield data in terms of unoccupied density of states is not straightforward because possible multiplet splitting of the resonant intermediate state, i.e., if the core-hole strongly interacts with the valence electrons, may lead to a complicated final-state structure. ${ }^{14}$ Previous experiments ${ }^{8}$ have shown, however, that for the late $4 d$ TM these effects are negligible and a simple interpretation of the yield curve in

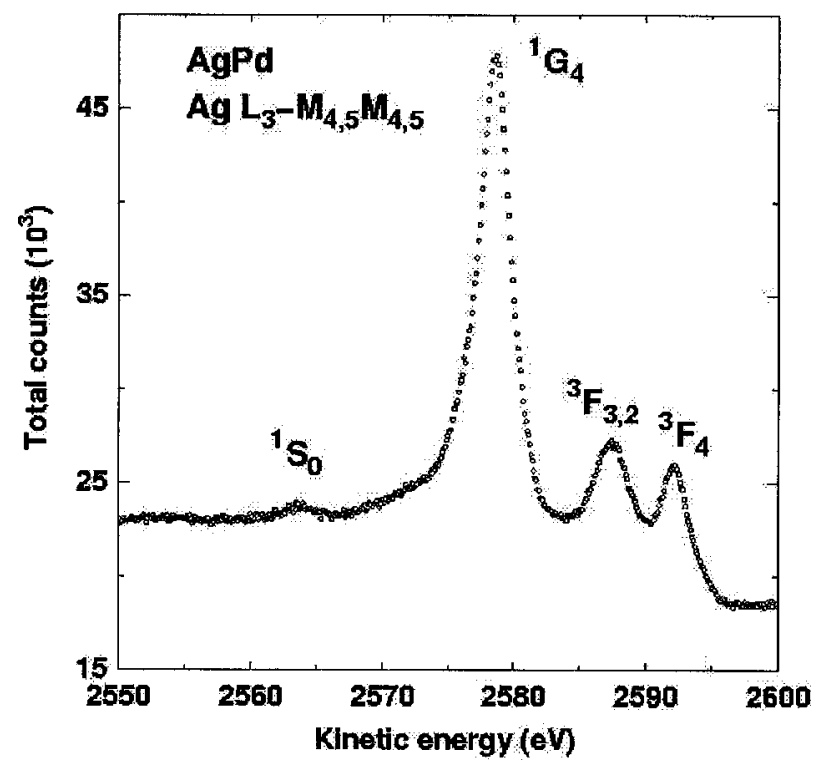

FIG. 1. Ag $L_{3} M_{4,5} M_{4,5}$ Auger spectrum of PdAg excited $3 \mathrm{eV}$ above the $L_{3}$ threshold. The most prominent peak corresponds to the ${ }^{1} G_{4}$ final state of the $3 d^{8}$ atomic multiplet structure, as indicated in the figure.

terms of one-electron DOS is justified.

The Ag $L_{3}$-edge XANES has been analyzed theoretically within a full multiple scattering framework. ${ }^{15,16}$ The detailed description of the computational scheme and the software package G4XANES has been published elsewhere. ${ }^{17}$ The crystal muffin-tin (MT) potential with touching MT spheres has

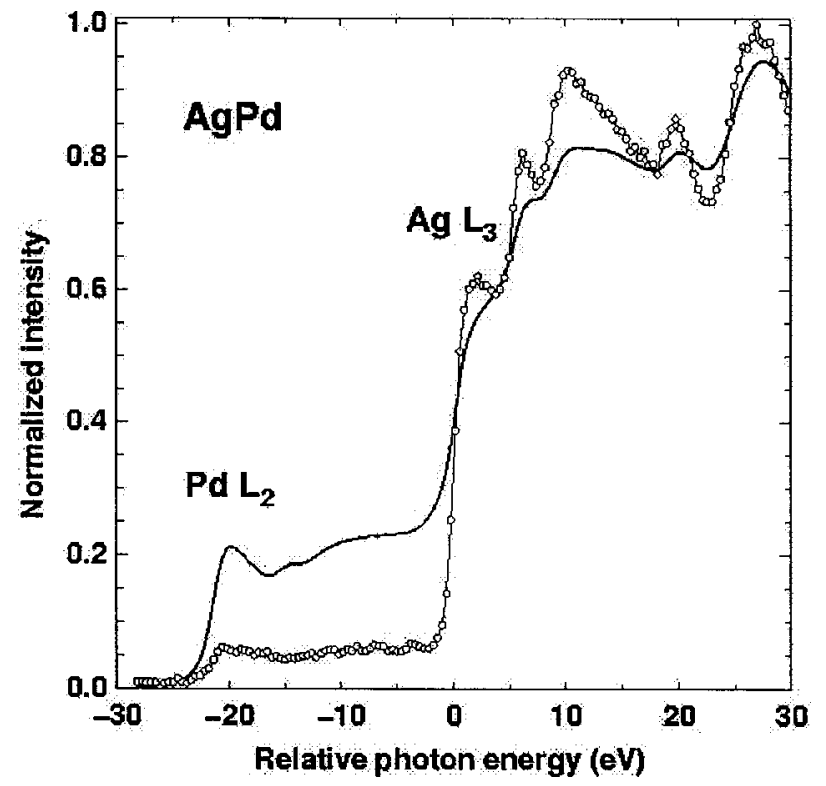

FIG. 2. Absorption spectra around the Ag $L_{3}$ edge in PdAg. The energy scale refers to the $\operatorname{Ag} L_{3}$ absorption threshold. In the total electron yield spectrum (solid line) there is a significant contribution of the underlying $\operatorname{Pd} L_{2}$ absorption structure. This background is reduced by a factor of 5 in the partial Ag Auger yield data (symbols). In addition, the spectral features are considerably enhanced because of the reduced core lifetime broadening. 
been constructed according to Mattheiss' prescription. ${ }^{18}$ Atomic charge densities were obtained with a self-consistent Dirac method. ${ }^{19}$ For Ag metal, a fcc structure with a lattice parameter of $4.0862 \AA$ was assumed. ${ }^{20}$ In a first approximation, the AgPd alloy was treated as having the same lattice symmetry but with a reduced average lattice constant of $3.9389 \AA$ A. Pd atoms are assumed to substitute half of the Ag sites in the fcc lattice, neglecting alloy fluctuation effects. ${ }^{11}$ The convergence of the calculated XANES shape was tested by successively increasing the number of shells, i.e., the number of atoms included into the cluster (not shown). It was found that for more than seven shells the change in the calculated spectrum is negligible. In the following, all calculations have been performed using a cluster of eight shells (123 atoms). Although the $\mathrm{Ag} L_{3}$-edge XANES is dominated by $p \rightarrow d$ dipole transitions (the $p \rightarrow d$ transition matrix element is at least a factor of 10 compared to that for $p \rightarrow s$ in the energy region of interest), both channels were taken into account for $L_{3}$-edge XANES calculation. The values of the MT sphere radii are $1.445 \AA$ ( $\mathrm{Ag})$ and $1.375 \AA(\mathrm{Pd})$. The MT constants (constant part of the averaged crystal potential, if vacuum level energy is zero) are $-13.35 \mathrm{eV}$ for the $\mathrm{Ag}$ site and $-12.94 \mathrm{eV}$ for the $\mathrm{Pd}$ site. In the calculation, phase shifts with orbital momentum up to 3 were included.

\section{RESULTS AND DISCUSSION}

In Fig. 3, theoretical and experimental results for the $\mathrm{Ag}$ $L_{3}$ XANES of AgPd alloy and Ag metal are presented. The calculation takes into account the experimental energy resolution (see above) and additional broadening due to the finite inelastic mean free path of the photoelectron which was modeled using an energy-dependent function. ${ }^{21}$ These factors were treated as contributions to the imaginary part of the self-energy. For better comparison, the Ag and AgPd spectra in Fig. 3 are normalized in intensity and aligned in energy position relative to peak $C$ of the XANES structure of Ag metal. The measured $L_{3}$-threshold energy of Ag metal, e.g., the inflection point of the leading absorption edge, is 3352.7 $\mathrm{eV}$ which is in accord with the $\mathrm{Ag} 2 p_{3 / 2}$ binding energy, $3352.9 \mathrm{eV}$, obtained by photoemission. ${ }^{22}$ It should be noted that the $\mathrm{Ag} L_{3}$ edge of $\mathrm{AgPd}$ is measured at $\sim 0.8 \mathrm{eV}$ lower photon energy relative to that of Ag metal. There is an overall good agreement between the measured and calculated absorption structures. The minimum above peak $C$ predicted by theory, however, is not observed in the experiment where the signal is rather flat. This might arise from additional inelastic damping of the photoelectrons, underestimated in the theory and disorder not accounted for in the model. In the threshold region, the intensity of the plateaulike shoulder $A$ is observed to decrease in AgPd relative to Ag metal. This behavior is also nicely reproduced in the calculation.

A starting point for the interpretation of these spectral changes in the $\mathrm{Ag} L_{3}$-XANES region of $\mathrm{AgPd}$ a few eV above $E_{F}$ is the difference in the nature of the $4 d \mathrm{TM}$ $L_{3}$-edge XANES for atoms with partially occupied (e.g., Pd) and fully occupied (e.g., Ag) $4 d$ levels. While the $\operatorname{Pd} L_{3}$ edge exhibits a well-known "white line" structure, $\mathrm{Ag} L_{3}$
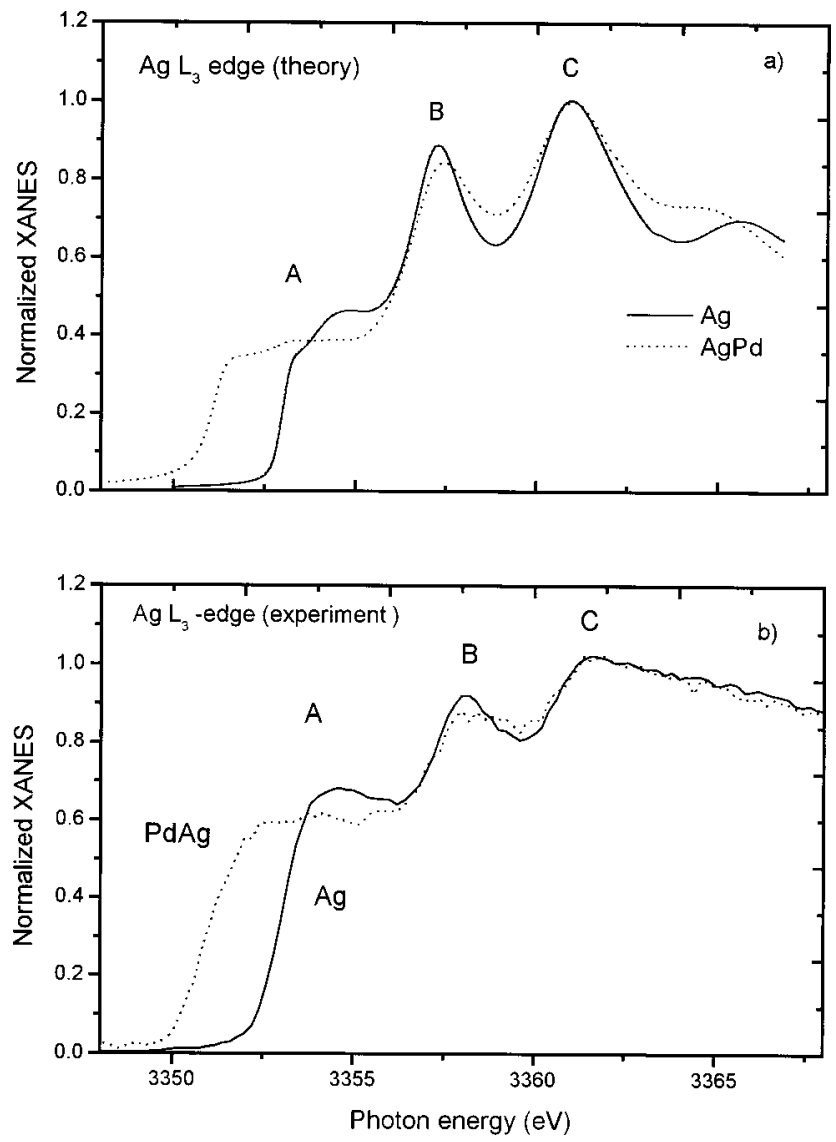

FIG. 3. Comparison of the theoretical full multiple-scattering results (a) and experimental data (b) for the $\mathrm{Ag} L_{3}$ XANES of AgPd alloy and $\mathrm{Ag}$ metal. The spectra are normalized in intensity and aligned in energy position to peak $C$ of the XANES structure in $\mathrm{Ag}$ metal.

XANES [as well as Au $L_{3}$ (Ref. 23)] does not. Since the Ag $4 d$ states are tighter bound and nominally fully occupied, they are more localized than $\mathrm{Pd} 4 d$ states. This results in different partial density of states at the Fermi level. The unoccupied states having Ag $d$ symmetry are no longer atomiclike $4 d$ states, but they are nearly free-electron states that are well described by Bloch wave function in the continuum. Accordingly, these states are not localized within the MT sphere of Ag, but delocalized in real space. On the contrary, $\mathrm{Pd} d$ states at the Fermi level are mainly atomiclike $4 d$ states, localized within the Pd MT sphere. Thus the analysis of the $\mathrm{Pd} L_{3}$ edge in the AgPd alloys provides information on the charge transfer of the relatively localized $\mathrm{Pd} 4 d$ electrons within the Pd MT sphere. On the other hand, the analysis of the $\mathrm{Ag} L_{3}$ edge is more challenging since the study of the Ag $L_{3}$ XANES provides information on the redistribution of nearly free-electron states of the conduction band just above the Fermi level. It is noteworthy that the creation of the core hole will localize the $4 d$ states somewhat but this effect should be the same for both the alloy and the metal.

In order to discuss the fine details of $\mathrm{Ag} d$ states distribution in the vicinity of the Fermi level, Fig. 4 displays the projected $d$ density of states including both the occupied and the unoccupied part. The calculation suggests that the density 


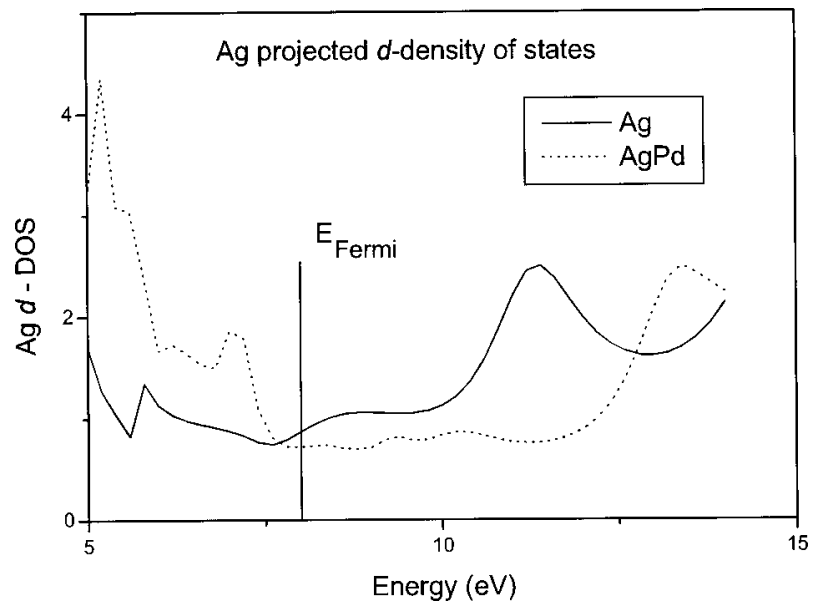

FIG. 4. The projected density of $\mathrm{Ag} d$ states close to the Fermi level including both occupied and unoccupied states of Ag metal and AgPd alloy.

of the occupied $\mathrm{Ag} d$ states well below the Fermi level increases during alloying (i.e., when $\mathrm{Pd}$ atoms replace $\mathrm{Ag}$ atoms in the Ag lattice) whereas the unoccupied density of states close to the Fermi level and about $4.5 \mathrm{eV}$ above it decreases, in agreement with the experimental data (cf. Fig. 3). Similar behavior has been observed in x-ray emission ${ }^{6}$ and theoretical calculations of $\mathrm{Ag}$ and $\mathrm{Pd}$ core level shifts. ${ }^{24}$ Because of the dipole selection rules, $\operatorname{Ag} L \beta_{2}$ fluorescence reflects the distribution of the occupied $\mathrm{Ag} d$ states in the

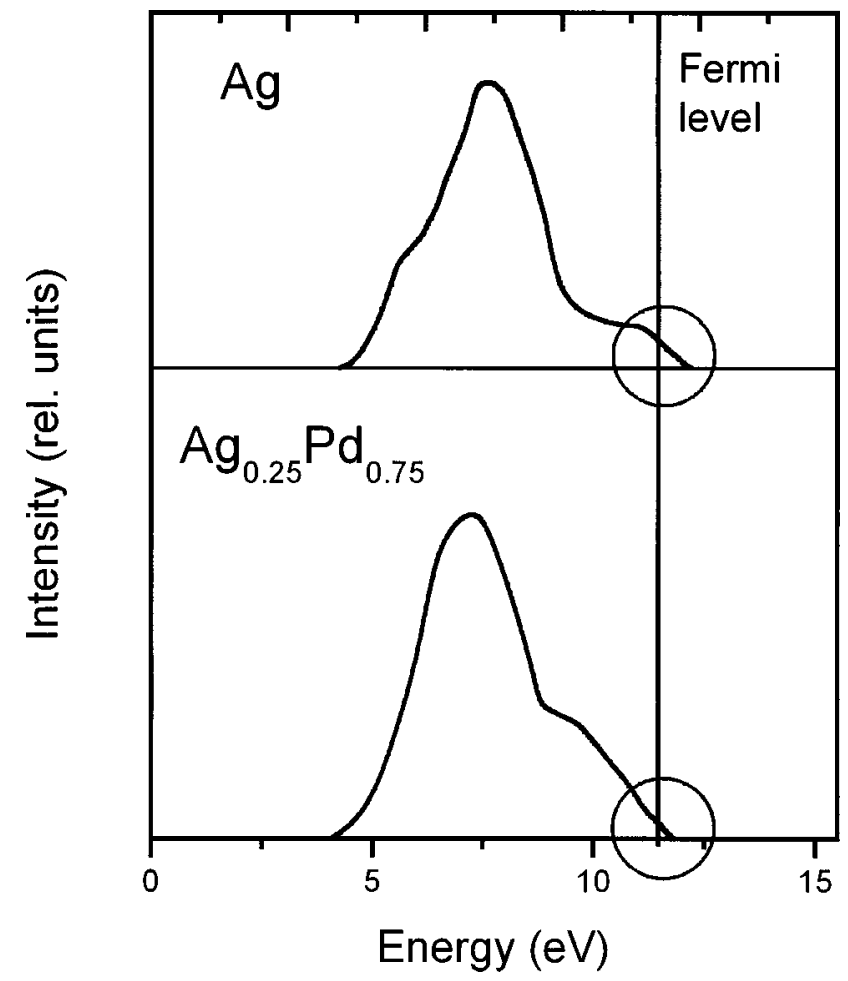

FIG. 5. $\mathrm{Ag} L \beta_{2}$ x-ray fluorescence spectra of $\mathrm{Ag}$ metal and $\mathrm{Ag}_{0.25} \mathrm{Pd}_{0.75}$ alloy corrected for the core-level width and experimental resolution (according to Ref. 6).
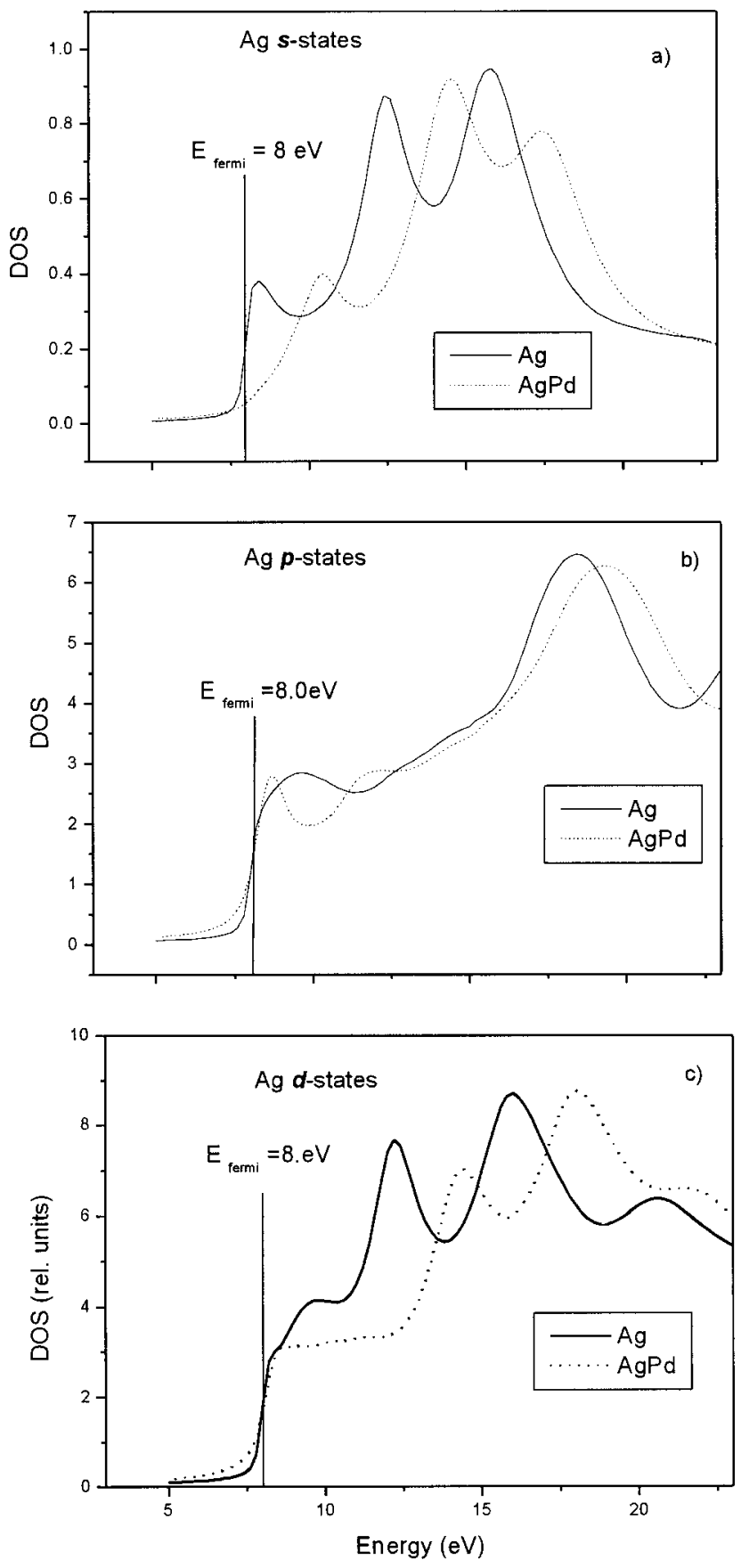

FIG. 6. Theoretical partial photoabsorption cross sections reflecting the distribution of unoccupied $\boldsymbol{s}, \boldsymbol{p}$, and $\boldsymbol{d}$ states in Ag metal and AgPd alloy up to $15 \mathrm{eV}$ above the Fermi level.

valence band. In Fig. 5, the $\mathrm{Ag} L \beta_{2}$ spectrum of Ag metal and $\mathrm{Ag}_{0.25} \mathrm{Pd}_{0.75}$ (from Ref. 6) shows a reduced intensity of the occupied states close to the Fermi level in the alloy relative to pure $\mathrm{Ag}$ metal, in accord with the calculation. The relative increase of the occupied $d$ DOS in the alloy is also consistent with the theoretical results obtained in Ref. 11.

In Fig. 6, we compare the partial photoabsorption cross sections reflecting the distribution of $\operatorname{Ag} s$ (panel $a$ ), $p$ (panel $b$ ), and $d$ (panel $c$ ) projected states just above the Fermi level 


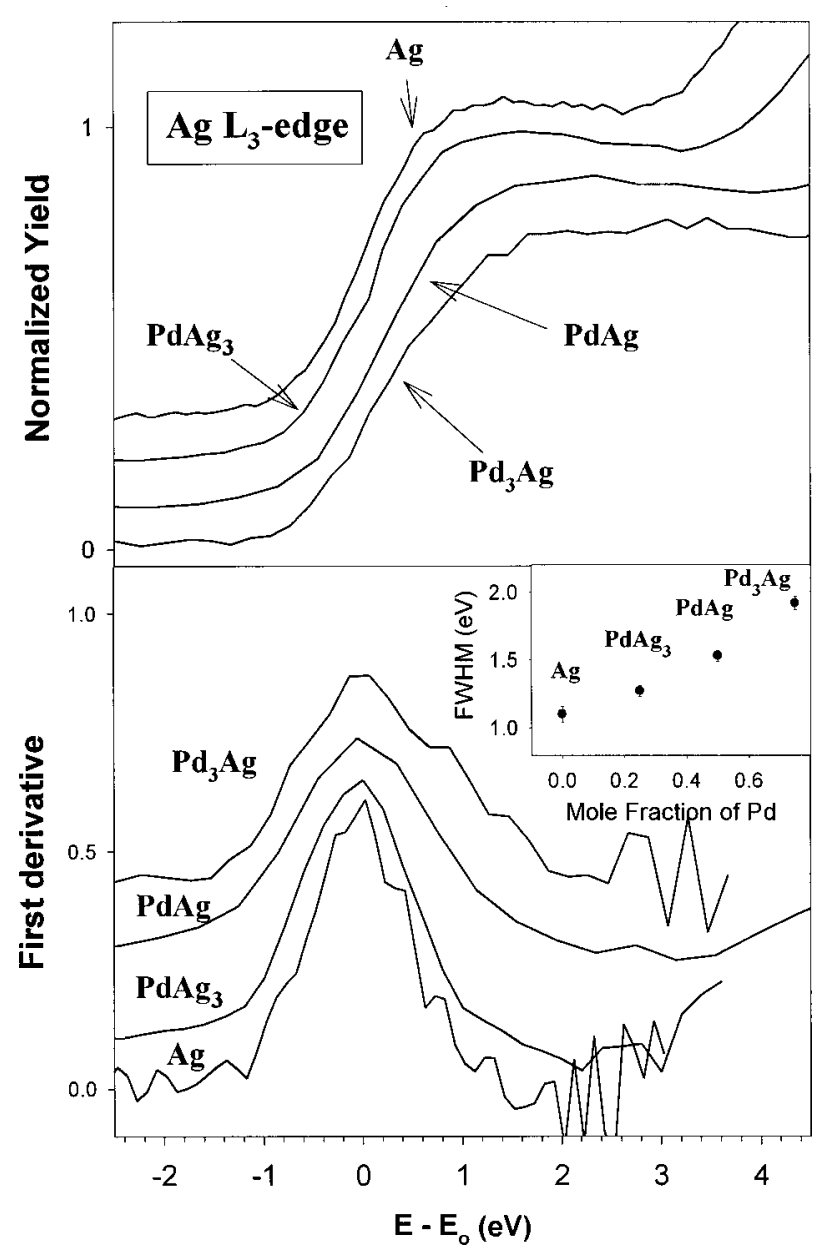

FIG. 7. $\mathrm{Ag} L_{3}$-edge jump profile of the Pd-Ag alloys. Top: spectra aligned at $E_{0}$ (point of inflection). Bottom: the corresponding first derivative; the full width at half maximum (FWHM) with error bars obtained from fitting is shown in the inset.

in $\mathrm{Ag}$ metal and $\mathrm{AgPd}$ alloy. Relatively little change occurs in the $p$-state distribution in the region around $1.5 \mathrm{eV}$ above $E_{F}$ where the $p$ DOS becomes more concentrated. Changes in the distribution of states having $d$ and $s$ symmetry are more pronounced. They are pushed towards higher energies upon alloying leading to a clear reduction of the DOS at the
Fermi level and several $\mathrm{eV}$ above. Note that the $s$-state contribution is small and has little effect on the $L_{3}$ XANES. The upward energy shift of the $\mathrm{Ag} s$ states could be related to a resonant interaction with the $\mathrm{Ag} p$ states, which are localizing above the Fermi level and therefore push the states of $s$ symmetry away from this interval. Such an interaction between the unoccupied states of different symmetry has been found previously in several compounds. ${ }^{25,26}$ Significant hybridization between occupied valence electron states in $\mathrm{Ag}-\mathrm{Pd}$ alloy has been found very recently. ${ }^{25}$

Finally, we note that charge redistribution upon alloying at the Fermi level is borne out in the edge jump profile (Fig. 7). By differentiating the edge jump and aligning the spectra at the threshold, we observe that the first derivative at the threshold exhibits a progressive broadening (also see the inset of Fig. 7) as Ag becomes more dilute in Pd. This observation indicates a small reduction (only noticeable in high resolution) in the $d$ densities of states at the Fermi level.

\section{CONCLUSIONS}

In summary, the redistribution of electronic states above the Fermi level of Ag metal and AgPd alloy was studied by high-resolution partial Auger-yield $\mathrm{Ag} L_{3}$ XANES in combination with a theoretical model employing a full multiplescattering approach. The agreement between experiment and theory is good, suggesting a reduction of the projected $\mathrm{Ag} d$ density of states just above the Fermi level upon alloy formation. It was shown that the $L_{3}$ XANES obtained with the present approach is a sensitive tool for the study of the redistribution of electronic states in the vicinity of the Fermi level. Since the fine structure of these states affects a large number of physical phenomena, this technique may also be applicable to the study of, e.g., low dimensional systems such as non-Fermi-liquid behavior in one-dimensional high$T_{c}$ superconductors and related systems (Luttinger liquids). ${ }^{27}$

\section{ACKNOWLEDGMENTS}

T.K.S. wishes to thank NSERC (Canada) for a Research Grant and NATO for a Collaborative Research Grant (CRG960384) for travel and conducting experiments at HASYLAB. A.V.S. is grateful to CCP (University of Western Ontario, Canada) for the support of present research.
${ }^{1}$ V. L. Moruzzi and C. B. Sommers, Calculated Electronic Properties of Ordered Alloys: A Handbook: The Elements and their $3 d / 3 d$ and $4 d / 4 d$ Alloys (World Scientific, New York, 1995), and references therein.

${ }^{2}$ S. Hüfner, Photoelectron Spectroscopy (Springer, Berlin, 1996), and references therein.

${ }^{3}$ Unoccupied Electronic States, edited by J. C. Fuggle and J. E. Inglesfield (Springer-Verlag, Berlin, 1992), and references therein.

${ }^{4}$ J. Hedman, M. Klasson, R. Nilsson, C. Nordling, M. F. Sorokina, O. I. Kljuslinkov, S. A. Nemnonov, X. X. Trapeznikov, and V. G. Zynjanov, Phys. Scr. 4, 195 (1971).
${ }^{5}$ I. Coulthard and T. K. Sham, Phys. Rev. Lett. 77, 4824 (1996).

${ }^{6}$ Yu. A. Babanov, O. B. Sokolov, and M. F. Sorokina, Phys. Status Solidi B 52, 155 (1972).

${ }^{7}$ T. K. Sham, Phys. Rev. B 31, 1888 (1985).

${ }^{8}$ W. Drube, R. Treusch, and G. Materlik, Phys. Rev. Lett. 74, 42 (1995).

${ }^{9}$ W. Drube, A. Lessmann, and G. Materlik, Jpn. J. Appl. Phys., Suppl. 32-2, 173 (1992).

${ }^{10}$ W. Drube, R. Treusch, T. K. Sham, A. Bzowski, and A. V. Soldatov, Phys. Rev. B 58, 6871 (1998).

${ }^{11}$ Z. W. Lu, S.-H. Wei, and Alex Zunger, Phys. Rev. B 44, 10470 (1991). 
${ }^{12}$ K. Berndt, U. Marx, and O. Brümmer, Phys. Status Solidi B 94, 541 (1979).

${ }^{13}$ K. Kokko, R. Laihia, M. Alatalo, P. T. Salo, M. P. J. Punkkinen, I. J. Väyrynen, W. Hergert, and D. Ködderitzsch, Phys. Rev. B 60, 4659 (1999).

${ }^{14}$ P. Carra, M. Fabrizio, and B. T. Thole, Phys. Rev. Lett. 74, 3700 (1995).

${ }^{15} \mathrm{P}$. Durham, in $X$-ray Absorption: Principle, Applications, Techniques of EXAFS, SEXAFS, XANES (Wiley, New York, 1989), p. 53.

${ }^{16}$ D. D. Vvedensky, D. K. Saldin, and J. B. Pendry, Comput. Phys. Commun. 40, 421 (1986).

${ }^{17}$ S. Della Longa, A. V. Soldatov, M. Pompa, and A. Bianconi, Comput. Mater. Sci. 4, 199 (1995).

${ }^{18}$ L. F. Mattheis, Phys. Rev. A 134, 970 (1964).

${ }^{19}$ J. P. Desclaux, Comput. Phys. Commun. 9, 31 (1975).
${ }^{20}$ R. W. G. Wyckoff, Crystal Structures (Interscience, New York, 1965).

${ }^{21}$ J. E. Müller, O. Jepsen, and J. W. Wilkins, Solid State Commun. 42, 365 (1982).

${ }^{22}$ W. Huschka, D. Ross, M. Maier, and E. Umbach, J. Electron Spectrosc. Relat. Phenom. 46, 273 (1988).

${ }^{23}$ C. C. Tyson, A. Bzowski, P. Kristof, M. Kuhn, R. Sammynaiken, and T. K. Sham, Phys. Rev. B 45, 8924 (1992).

${ }^{24}$ I. A. Abrikosov, W. Olovsson, and B. Johansson, Phys. Rev. Lett. 87, 176403 (2001).

${ }^{25}$ A. V. Soldatov, T. S. Ivanchenko, S. Della Longa, A. Kotani, Y. Iwamoto, and A. Bianconi, Phys. Rev. B 50, 5074 (1994).

${ }^{26}$ A. V. Soldatov, M. Kasrai, and G. M. Bancroft, Solid State Commun. 115, 687 (2000).

${ }^{27}$ D. K. K. Lee and Y. Chen, Phys. Rev. Lett. 69, 1399 (1992). 